\title{
Exploring societal preferences for energy sufficiency measures in Switzerland
}

\section{OPEN ACCESS}

Edited by:

Tobias Brosch,

University of Geneva, Switzerland

Reviewed by:

Anya Skatova,

University of Nottingham, UK

Andrea Tabi,

University of St. Gallen, Switzerland

*Correspondence:

Corinne Moser,

Institute of Sustainable Development,

School of Engineering, Zurich

University of Applied Sciences,

Technoparkstrasse 2, Winterthur

8401, Switzerland

corinne.moser@zhaw.ch

Specialty section:

This article was submitted to Energy Systems and Policy, a section of the journal Frontiers in Energy Research

Received: 15 December 2014 Accepted: 25 August 2015 Published: 16 September 2015

Citation:

Moser C, Rösch A and Stauffacher M (2015) Exploring societal preferences

for energy sufficiency measures in

Switzerland.

Front. Energy Res. 3:40.

doi: 10.3389/fenrg.2015.00040

\begin{abstract}
Corinne Moser ${ }^{1,2 *}$, Andreas Rösch ${ }^{2}$ and Michael Stauffacher ${ }^{2,3}$
${ }^{1}$ Institute of Sustainable Development, School of Engineering, Zurich University of Applied Sciences, Winterthur, Switzerland, ${ }^{2}$ Natural and Social Science Interface, Institute for Environmental Decisions, Department of Environmental Systems Science, ETH Zürich, Zürich, Switzerland, ${ }^{3}$ Transdisciplinarity Laboratory, Department of Environmental Systems Science, ETH Zürich, Zürich, Switzerland
\end{abstract}

Many countries are facing a challenging transition toward more sustainable energy systems, which produce more renewables and consume less energy. The latter goal can only be achieved through a combination of efficiency measures and changes in people's lifestyles and routine behaviors (i.e., sufficiency). While research has shown that acceptance of technical efficiency is relatively high, there is a lack of research on societal preferences for sufficiency measures. However, this is an important prerequisite for designing successful interventions to change behavior. This paper analyses societal preferences for different energy-related behaviors in Switzerland. We use an online choice-based conjoint analysis $(N=150)$ to examine preferences for behaviors with high technical potentials for energy demand reduction in the following domains: mobility, heating, and food. Each domain comprises different attributes across three levels of sufficiency. Respondents were confronted with trade-off situations evoked through different fictional lifestyles that comprised different combinations of attribute levels. Through a series of trade-off decisions, participants were asked to choose their preferred lifestyle. The results revealed that a vegetarian diet was considered the most critical issue that respondents were unwilling to trade off, followed by distance to workplace and means of transportation. The highest willingness to trade off was found for adjustments in room temperature, holiday travel behaviors, and living space. Participants' preferences for the most energy-sufficient lifestyles were rather low. However, the study showed that there were lifestyles with substantive energy-saving potentials that were well accepted among respondents. Our study results suggest that the success of energy-sufficiency interventions might depend strongly on the targeted behavior. We speculate that they may face strong resistance (e.g., vegetarian diet). Thus, it seems promising to promote well-balanced lifestyles, rather than extremely energy-sufficient lifestyles, as potential role models for sufficiency.

Keywords: energy, sufficiency, societal preferences, routine behavior, lifestyles, conjoint analysis 


\section{Introduction}

\section{The Importance of Energy Sufficiency for Switzerland's Energy Transition}

Countries worldwide are facing challenging transitions of their energy systems with regard to fighting climate change and declining availability of fossil fuels. Switzerland has adopted a new energy strategy (Energy Strategy 2050) that promotes the implementation of new renewables, the stepwise phase-out of nuclear power, and sets ambitious reduction targets for per capita energy consumption (Swiss Federal Council, 2013). This goal shall be achieved primarily through increased energy efficiency, i.e., through the implementation of technologies that require less energy to maintain current levels of services. Examples of such energy-efficient technologies include cars that use less fuel per kilometer and well-insulated buildings that require less heat.

However, there are technological and economical limitations to energy efficiency. Furthermore, increased energy efficiency often causes rebound effects (Herring, 2006; Darby, 2007), which at least partly offset the saved resources (e.g., energy, time, money). For example, although many appliances, such as fridges or TVs, are more energy efficient than ever before, these appliances have also increased in size and/or in number over time. Along these lines, researchers have also found that people rely on symbols of energy efficiency, which may lead to paradoxical effects (Sütterlin and Siegrist, 2014). For example, in an experiment, participants judged a person driving an energy-efficient car (i.e., a Prius) over longer distances to be more energy conscious than an SUV driver who covered shorter distances - and so, in total, consumed less energy than the Prius driver (Sütterlin and Siegrist, 2014).

Thus, in order to guarantee an absolute reduction in energy consumption, efficiency needs to be complemented by more sustainable consumption patterns. This requires behavioral changes on the part of energy consumers. This perspective was confirmed by a recent study in which Notter et al. (2013) estimated Switzerland's potential to become a $2000-\mathrm{Watt} / 1$ ton $\mathrm{CO}_{2}$ society ${ }^{1}$. The authors conclude that this goal is only realistic "when assuming a pronounced technological increase in efficiency combined with a smart sufficiency strategy" (Notter et al., 2013, p. 4019).

Sufficiency can be understood as a process of changing existing consumption patterns for more sustainable ones. The literature distinguishes two different approaches to sufficiency (for an overview, see Jenny, 2014). First, in a narrow sense, sufficiency can be understood as a necessary complement to energy efficiency and renewable energy sources in order to reach political goals regarding climate targets, resource use, or per capita energy consumption. Second, it can also be understood as a critique of our consumer society and our growth-based economic system, as well as of respective attempts to change these systems (Linz et al., 2002; Linz, 2012). In this study, we focus on the former, narrower understanding of sufficiency: that is, while energy efficiency refers to technological means to minimize resource input, energy

\footnotetext{
${ }^{1}$ The 2000-Watt/1 ton $\mathrm{CO}_{2}$ society is a Swiss energy vision that envisages a more equal distribution of global energy consumption by setting per capita consumption goals, it is very popular among Swiss authorities and academics (http:// www.2000watt.ch).
}

sufficiency refers to changes in individual behaviors that lead to lower demand for energy services. In accordance with Breukers et al. (2013), we understand energy-sufficient behavior to involve changes in routine behaviors and lifestyles that lead to lower energy consumption. Examples of energy-sufficient behaviors include line-drying laundry instead of using a tumble dryer, eating vegetarian food instead of meat, commuting by bike instead of by private car, and so on.

With respect to private energy consumption in Switzerland, over the entire lifecycle of products and services, the domains of mobility, heating, and food are the most energy-demanding (Notter et al., 2013). A study by Jungbluth and Itten (2012) indicated substantial potential for energy-sufficient behaviors in these domains. In their study, the following reduction potentials in primary energy consumption were found: nutrition at around $8 \%$ (i.e., eating vegetarian and seasonal food); mobility at around 17\% (traveling by bike/walking) or around 11\% (traveling by public transport); and living at around $12 \%$ (i.e., lowering the room temperature, reducing the living space per person).

To unlock these sufficiency potentials, private consumers are key agents of change. However, private energy consumption patterns are strongly shaped by habits, norms, and cultural, social, and technological contexts and are, therefore, difficult to change (e.g., Owens and Driffill, 2008). Under certain conditions, interventions have the potential to induce such changes (Thøgersen, 2005).

\section{Background: Private Energy-Saving Behaviors and Interventions to Change Behavior}

There exists a broad range of research that empirically tests or reviews interventions to change energy-relevant behaviors in different national contexts (Abrahamse et al., 2005; Steg and Vlek, 2009; Mourik and Rotmann, 2013). Steg and Vlek (2009) suggested a general framework for encouraging pro-environmental behavior, which is also relevant in the context of promoting energy-sufficient behavior. To design successful interventions, it is important to identify the relevant behaviors to be changed and to understand how they are influenced. This means that (i) those behaviors that actually have an impact on energy consumption should be identified (Gardner and Stern, 2008; Huddart Kennedy et al., 2015) and (ii) there is a need to better understand "the feasibility of various behavior changes and the acceptability of its consequences" (Steg and Vlek, 2009, p. 310). While (i) can be assessed from a technical perspective, (ii) requires a thorough understanding of people's current energy-saving behavior and their preferences regarding behavior change. In other words, a purely technical approach is not enough to design a successful intervention; it needs to be combined with social-scientific knowledge on behavior and behavior change.

Many studies in Switzerland and internationally that take a social-scientific perspective on energy saving differentiate between curtailment behaviors and efficiency decisions (Gardner and Stern, 2008; Karlin et al., 2012). As the topic of this study is energy sufficiency, we focus on the former aspect. A representative survey in Switzerland analyzed Swiss people's current energysaving behaviors in the domains of housing, food, and mobility (Sütterlin et al., 2011). People on average perform energy-saving behaviors in the domain of housing very often (e.g., turning the 
TV off when not watching it, filling the washing machine to its capacity, ventilating briefly but intensely during winter). By contrast, energy-saving behaviors in the domain of food (e.g., avoiding buying foods that are flown in, buying seasonal fruits and vegetables) and, in particular, energy-saving behaviors in the domain of mobility (e.g., going on holidays by train, covering short distances by foot or bicycle) are performed less frequently on average (see Table 1 in Sütterlin et al., 2011). This study did not cover meat consumption, although this is a crucial factor with respect to energy consumption in the domain of food (Dutilh and Kramer, 2000). The results of this Swiss study are in line with those of a Dutch study (Poortinga et al., 2003) that analyzed the acceptability of different energy-saving measures and found that such behaviors as switching off the lights or appliances are well accepted, while such behaviors as going on holidays by train or altering food patterns were somewhat contested. A study in nine OECD countries focusing on housing identified that turning off lights in unused rooms and fully loading washing machines and dishwashers were the most commonly performed energy-saving behaviors, while switching off stand-by modes in appliances seemed less popular (Urban and Ščasný, 2012). The reported results $^{2}$ indicate that private energy-saving behavior is domain dependent and, similarly, that people's preferences for energysaving measures differ for different domains.

What is more, there is a need to better understand contextual influences on energy consumption to design successful interventions (Steg and Vlek, 2009). Barr et al. (2011) noted with some criticism that studying and promoting sustainable consumption is often focused on isolated behaviors in the everyday "home" context where many pro-environmental behaviors (e.g., turning off lights) are socially desired and require no or only small adjustments in lifestyle. With changing contexts, for example when traveling to holidays, pro-environmental behaviors (e.g., not flying to a distant country) often ask for substantial adjustments in lifestyle that leads to conflicts and trade-offs. From focus group discussions, the authors conclude that, "in short, holidays were 'off limits' to sustainability” (Barr et al., 2011, p. 717). This means that designing successful interventions that actually have an impact on energy consumption requires a comprehensive approach that takes into account different contexts where energy is consumed, such as the home, in transit (e.g., from home to work), and while traveling (e.g., on vacation). At the same time, behavior in everyday situations (e.g., commuting) as well as in extraordinary situations (e.g., traveling to holidays) should be considered.

\section{Goal of this Study}

The literature review has revealed that (i) for realizing the energy transition in Switzerland, private consumers are key agents of change, (ii) there exist considerable energy-saving potentials through more energy-sufficient behavior, (iii) appropriate interventions may help unlock these potentials, (iv) an important prerequisite for designing successful interventions is knowing

\footnotetext{
${ }^{2}$ While the cited papers all use a quantitative approach, there are various studies on the issue of energy consumption that use more qualitative approaches, such as focus groups (e.g., Barr et al., 2011) or ethnographic research (e.g., Higginson et al., 2014).
}

what behaviors have the most impact on energy consumption, how they are influenced by context and what people's preferences are regarding behavior change. While many studies exist that analyze current energy-saving behaviors in Switzerland and in other countries, our study focuses on people's preferences for behaviors that differ in energy-sufficiency. Our approach considers behaviors that have a considerable impact on private energy consumption. Furthermore, behaviors are evaluated together as lifestyles, which are characterized by certain behavior patterns in different domains (everyday mobility, holiday travel, housing, food consumption). Thereby, different contexts for energy-sufficient behavior are considered. In other words, we aim to analyze what people think about energy sufficiency in different domains of life and to which energy-sufficient behaviors they can relate.

The goal of this paper is to identify societal preferences in Switzerland concerning different energy-related behaviors in order to reveal barriers and opportunities related to the promotion of energy sufficiency. Such knowledge provides an important basis for designing successful energy-sufficiency interventions by identifying potential levers and "no-go" areas for such interventions. More concretely, we investigate the following research questions:

- Which energy-sufficiency-related domains and behaviors do people prefer when evaluating different lifestyles?

- Which energy-sufficient lifestyles are perceived to be attractive by the public?

\section{Context Information about Switzerland}

As our analysis is focused on Switzerland, we briefly provide some key figures on private energy consumption as well as some context information about the domains we are looking at, that is, commuting, holiday travel, housing, and meat consumption. Swiss households demand $29 \%$ of final energy - mostly for heating and hot water - and mobility/transport demands 35\% (Swiss Federal Office of Energy, 2014). In the domain of mobility and transport, $74 \%$ of final energy is demanded for transporting people on the road, that is, mostly for private mobility (Swiss Federal Office of Energy, 2013). Although Switzerland has an excellent public transport system, approximately half of the inhabitants own a car (536 cars per 1000 inhabitants in 2014; Swiss Federal Statistical Office, 2015d). However, the level of motorization is usually lower in bigger cities compared to rural areas. On average, Swiss commuters commute $14.3 \mathrm{~km}$ from home to the workplace (one way). Of these commuters, $53 \%$ commute by car, $30 \%$ use public transport (train, tram, and bus), 9\% bike, and 6\% walk (Swiss Federal Statistical Office, 2014). In 2012, Swiss people (older than 6 years) completed a total of 20,300,000 trips with at least one overnight stay, which is roughly three trips per person. The purpose of $65 \%$ of these trips was holidays, which people spend abroad ( $2 / 3$ of cases, $1 / 3$ in Switzerland). Around $50 \%$ of trips were made by car and $27 \%$ by plane (Swiss Federal Statistical Office, 2013b). The average living space per capita was $45 \mathrm{~m}^{2}$ in 2013 . Around $60 \%$ of Swiss people rent their home, while around $40 \%$ are homeowners (Swiss Federal Statistical Office, 2015a). In 2012, around 25\% of Swiss people ate meat almost every day (6-7 days per week); around 50\% ate meat 3-5 days per week, around $20 \%$ ate meat $1-2$ times a week, and $3 \%$ never ate meat (Swiss Federal Office of Public Health, 2014). 


\section{Materials and Methods}

\section{Conjoint Analysis}

We apply a conjoint analysis to determine societal preferences for different fictional lifestyles that are characterized by different levels of energy-sufficient behaviors in relevant domains. Conjoint analysis is a method for studying complex decisions that are characterized by trade-offs among different attributes. This method has classically been used in consumer and marketing research (Green and Srinivasan, 1978) and has recently been applied to energy and infrastructure-related decisions (e.g., Dohle et al., 2010; Krütli et al., 2012; Rudolf et al., 2014). Participants are confronted with decision situations composed of sets of attributes. For example, decisions regarding future energy systems may be characterized by different prices and production technologies. Each attribute is associated with different levels (e.g., levels for price: different prices per kilowatt hour of electricity; levels of energy production technologies: solar, nuclear, wind, and hydropower). Participants are then asked to evaluate the decision situation by providing rankings, which requires them to consider combinations of different attribute levels jointly to make a decision. Next, the relative importance values of the different attributes and the part-worth utilities of all the levels can be assessed.

An advantage of conjoint analysis is that it reflects real-world decisions, which are usually characterized by combinations of criteria. Furthermore, it measures preferences indirectly, thus minimizing the potential for respondents to give socially desired responses (Sattler and Hensel-Börner, 2001). From a methodological perspective, another advantage is that not all combinations of levels need to be evaluated empirically; instead, utilities of all combinations can be estimated based on a limited set of choices.

In this study, a choice-based conjoint ( $\mathrm{CBC}$ ) was applied. The main difference between a $\mathrm{CBC}$ and other conjoint procedures is that, in a $\mathrm{CBC}$, rather than ranking or rating different options, participants choose their preferred option (Sawtooth Software, 2008). For assessing choices at an individual level, a hierarchical Bayesian estimation was applied.

\section{Attributes and Levels}

For the study at hand, only domains with high-energy-saving potentials were chosen (based on Jungbluth and Itten, 2012; Notter et al., 2013). Specifically, we selected the domains of mobility, heating, and food. For each of these domains, a set of attributes was selected:

- Mobility: distance to workplace, means of transport when commuting, holiday travel behavior.

- Heating: amount of heated living space per person, room temperature.

- Food: weekly meat consumption.

The basis for selecting these attributes was a study by Notter et al. (2013), who analyzed and quantified private behaviors based on their cumulative energy demand (CED), global warming potential (GWP) and environmental impact (EI99) by considering the entire lifecycles surrounding these behaviors. Private car use was the most important influencing factor for all three indicators (38\% of CED, $31 \%$ of GWP, and $29 \%$ of EI99). Additional important influencing factors were heating (26\% of CED, $25 \%$ of GWP, and 18\% of EI99) and food (6\% of CED, 15\% of GWP, and 20\% of EI99). Private aviation accounted for $7 \%$ of CED, 5\% of GWP, and 6\% of EI99 (data based on Notter et al., 2013). Also Jungbluth and Itten (2012) identified substantial reduction potentials for primary energy consumption in the domains of mobility, living, and nutrition.

For each attribute, three different levels were defined based on the literature or on thorough discussions among the authors of this paper, such that Level 1 is set as the least energy-sufficient level and Level 3 is the most energy-sufficient level. In contrast to most existing research, this research makes no explicit reference to energy consumption in its descriptions of lifestyles. Rather, the focus is on concrete social practices, which seems to be a more appropriate measurement for the embedded character of energy consumption. Furthermore, this approach also serves the purpose of describing lifestyles realistically, without the use of extensive technical jargon. Table 1 provides an overview of the selected domains, attributes, and levels.

\section{Design of Conjoint Analysis and Procedure}

The defined attributes and levels served as a basis to describe fictional characters and their lifestyles. These lifestyles were composed randomly by combining different levels (one per attribute, full-profile $\mathrm{CBC}$ ). In each decision situation, participants were presented with three different lifestyles and then asked to choose their preferred lifestyle (see Table 2). The study used a full-profile design, meaning that all attributes (with different levels each time) were represented in every option. For each option, the sequence of attributes was kept constant in order to maintain consistency and to better enable comparisons of levels across options. The study was a forced-choice situation; that is, there was no possibility to not choose an option.

Each participant made 10 choices in total (i.e., 10 decision situations): eight randomized tasks and two fixed holdout tasks (all participants evaluated the same two holdout tasks). The holdout tasks were used to validate the conjoint model (Orme et al., 1997; see Chapter Model Fit). Sawtooth Software was used to conduct the experiments and analyze the results (Sawtooth Software, 2008). Three sample lifestyles are presented in Table 2.

The data were collected as part of the second author's master's thesis (Rösch, 2013) in autumn of 2013 in the German-speaking part of Switzerland. Participants were recruited from an online panel and received a small incentive for participation. Potential participants were invited to the study by e-mail. The participants first responded to the $10 \mathrm{CBC}$ tasks described above. Afterwards, they answered questions on their personal energy-related behaviors, as well as socio-demographic questions. On average, participants required 12.5 min to complete the survey. All participants who completed the survey were included in the statistical analyses.

\section{Sample}

In total, $N=150$ participants took part in the study. On average, the participants were 47.7 years old ( $S D=12.67$ years), with youngest participant being 18 and the oldest being 66 years old. $52 \%(n=78)$ of respondents were female. A total of $50 \%$ 
TABLE 1 | Domains, attributes, and levels for the conjoint analysis.

\begin{tabular}{|c|c|c|c|}
\hline Domain & Attribute & Level & Level description \\
\hline \multirow[t]{9}{*}{ Mobility } & Distance to workplace (Swiss & 1 & $100 \mathrm{~km}$ from home to workplace (100 km) \\
\hline & Federal Statistical Office, 2012) & 2 & $10 \mathrm{~km}$ from home to workplace $(10 \mathrm{~km})$ \\
\hline & & 3 & $2 \mathrm{~km}$ from home to workplace $(2 \mathrm{~km})$ \\
\hline & Means of transport & 1 & Car or motorcycle (car) \\
\hline & & 2 & Public transport or park and rail (public transport) \\
\hline & & 3 & Public transport or bike (public transport/bike) \\
\hline & Holiday travel behavior & 1 & Short trips in Europe, vacations on another continent, solely air travel (World) \\
\hline & & 2 & $\begin{array}{l}\text { Short trips to cities in adjacent countries, vacations within Europe, air travel for } \\
\text { vacations, trains for short trips (Europe) }\end{array}$ \\
\hline & & 3 & $\begin{array}{l}\text { Short trips and vacations in Switzerland or adjacent countries, train whenever possible } \\
\text { or car otherwise (Switzerland) }\end{array}$ \\
\hline \multirow[t]{6}{*}{ Heating } & Living space (Swiss Federal & 1 & $60 \mathrm{~m}^{2}$ per person $\left(60 \mathrm{~m}^{2}\right)$ \\
\hline & Statistical Office, 2015a) & 2 & $50 \mathrm{~m}^{2}$ per person $\left(50 \mathrm{~m}^{2}\right)$ \\
\hline & & 3 & $40 \mathrm{~m}^{2}$ per person $\left(40 \mathrm{~m}^{2}\right)$ \\
\hline & Room temperature & 1 & T-shirt can be worn even if cold outside (high) \\
\hline & (Stadt Zürich, 2006) & 2 & Thin pullover and trousers are worn if cold outside (medium) \\
\hline & & 3 & Thick pullover and warm socks are worn if cold outside (low) \\
\hline \multirow[t]{3}{*}{ Food } & Weekly meat consumption & 1 & Meat at least once a day (daily) \\
\hline & (Notter et al., 2013) & 2 & Meat 3-4 times a week (3-4 times) \\
\hline & & 3 & Vegetarian or vegan diet (never) \\
\hline
\end{tabular}

Terms in brackets indicate the short labels for the levels.

TABLE 2 | Exemplary description of three lifestyles, as presented in the study.

The lifestyles of different people are presented below. Please read through them carefully and choose the lifestyle that appeals to you the most. Click the respective button at the end. Even if it is difficult for you to choose, please select one. (1 of 10 decisions)

Work $\quad$ The person lives in Zürich and works in Bern. He or she commutes daily (100 $\mathbf{~ k m}$ one way). He or she would consider moving if a new job involved a commute of more than $130 \mathrm{~km}$ each way (e.g., Zürich-Fribourg)

He or she commutes to work by public transport or park and rail

Travel The person regularly goes on short trips within Europe (e.g., a weekend trip to Rome, London, or Barcelona). At least once a year, he or she travels to another continent for vacation (e.g., Maldives, the USA, or Brazil). For short trips and longer vacations, he or she usually takes the plane

Housing The person's flat offers $\mathbf{5 0} \mathbf{~ m}^{\mathbf{2}}$ per person The colder it gets, the more clothes he or she wears at home to keep warm. On days that are particularly cold, he or she wears thick clothes and warm socks

Food The person consumes meat three to four times per week
The person lives and works in Zürich. His or her place of work is $\mathbf{2} \mathbf{~ k m}$ away from home. $\mathrm{He}$ or she would consider moving if a new job involved a commute of more than $10 \mathrm{~km}$ each way

He or she commutes to work by car

The person regularly goes on short trips within Switzerland (e.g., a weekend trip to Ticino or to the Alps). He or she spends vacations in Switzerland or in adjacent countries (e.g., France or Germany). For short trips and longer vacations, he or she usually uses public transport whenever possible or car otherwise

The person's flat offers $\mathbf{4 0} \mathbf{~ m}^{\mathbf{2}}$ per person Even if it is less than $0^{\circ} \mathrm{C}$ outside, he or she only wears thin clothes at home because the rooms are comfortably warm

The person consumes meat daily
The person lives in Zürich and works in Thalwil. $\mathrm{He}$ or she commutes daily (10 $\mathbf{~ k m}$ one way). He or she would consider moving if a new job involved a commute of more than $50 \mathrm{~km}$ each way (e.g., Zürich-Olten)

He or she commutes to work by public transport or park and rail

The person regularly goes on short trips in adjacent countries (e.g., a weekend trip to Paris or Berlin). At least once a year, he or she travels to a more distant country in Europe (e.g., Spain or Norway) or to a close country on another continent (e.g., Egypt). For vacation, he or she takes a plane, and for short trips, he or she uses public transport

The person's flat offers $\mathbf{6 0} \mathbf{~ m}^{\mathbf{2}}$ per person

Even if it is less than $0^{\circ} \mathrm{C}$ outside, he or she only wears thin clothes at home because the rooms are comfortably warm

The person does not eat meat; he or she is a vegetarian or vegan

$\bigcirc$

Bolded phrases were depicted in red, and the original descriptions were in German.

of participants had concluded vocational training, $20 \%$ had completed higher education (e.g., university, $\mathrm{PhD}$ ), 16\% had completed senior high school, $5 \%$ had completed higher vocational training, $4 \%$ had completed compulsory school, and the rest did not specify their education level.

Regarding political attitudes, most participants positioned themselves in the center of a left wing-right wing scale [from 1 (left) to 7 (right); $M=3.98, S D=1.13$ ]. Most participants (43\%) lived in two-people households, $24 \%$ lived in single-person households, $16 \%$ lived in three-people households, and the remainder lived in households larger than three people.

Table 3 summarizes key characteristics of our sample and compares them to Swiss average data (where comparable Swiss data are available; Swiss Federal Statistical Office, 2013a, 2015b,c). 
TABLE 3 | Key characteristics of our sample in comparison with Swiss population statistics.

\begin{tabular}{|c|c|c|}
\hline Key characteristics & Study sample $(N=150)$ & Swiss population \\
\hline Gender & $52 \%$ females, $48 \%$ males & 51\% females, 49\% males (Swiss Federal Statistical Office, 2015c) \\
\hline Age (mean) & 47.7 years & 41.8 years (Swiss Federal Statistical Office, 2015c) \\
\hline Education & $\begin{array}{l}50 \% \text { vocational training } \\
20 \% \text { higher education (e.g., university, PhD) } \\
16 \% \text { senior high school } \\
5 \% \text { higher vocational training } \\
4 \% \text { compulsory school } \\
\text { Rest: other }\end{array}$ & $\begin{array}{l}44 \% \text { vocational training } \\
18 \% \text { higher education (e.g., university, PhD) } \\
9 \% \text { senior high school } \\
13 \% \text { higher vocational training } \\
15 \% \text { compulsory school } \\
\text { (Swiss Federal Statistical Office, 2013a) }\end{array}$ \\
\hline Household size & $\begin{array}{l}\text { 24\% one person } \\
43 \% \text { : two people } \\
\text { 16\%: three people } \\
\text { Rest: larger households }\end{array}$ & $\begin{array}{l}\text { 35\% one person } \\
\text { 33\%: two people } \\
\text { 13\%: three people } \\
\text { Rest: larger households } \\
\text { (Swiss Federal Statistical Office, 2015b) }\end{array}$ \\
\hline
\end{tabular}

The sample is approximately representative of Switzerland's population with regard to gender. With respect to vocational and university education, the sample is roughly comparable to the Swiss population, though, in our sample, fewer people had completed only compulsory school and fewer people had completed higher vocational training than the Swiss average. Regarding age, our sample is slightly older than the Swiss average; however, this could be due to the fact that only participants 18 or older were invited to participate in the survey. Regarding household size, more people in our sample lived in two-people households, and fewer people lived in single households than in the Swiss population.

Finally, participants were asked about their personal behaviors in relation to their energy consumption in the domains of mobility, housing, and food. Around 65\% of participants lived $10 \mathrm{~km}$ away from their workplace or closer, around 30\% lived between 10 and $50 \mathrm{~km}$ from their workplace, and around 5\% lived more than $50 \mathrm{~km}$ from their workplace. Most participants (41\%) used their car to commute to work, 35\% used public transport, $9 \%$ used bikes, and $15 \%$ walked to their workplace. Over the last 5 years, participants had flown, on average, around nine times $(M=9.33, S D=10.84)$. In terms of living space, $34 \%$ of participants used $40 \mathrm{~m}^{2}$ per person or less, $25 \%$ of participants used around $50 \mathrm{~m}^{2}$ per person, $19 \%$ used around $60 \mathrm{~m}^{2}$ per person, and the remainder used more than $60 \mathrm{~m}^{2}$ per person. Participants also indicated the general room temperature they used during the heating season in their apartments: around 33\% of participants wore only light clothing during the winter time, $37 \%$ wore thin pullovers and trousers, and $30 \%$ wore thick clothes and warm socks. With regard to food consumption patterns, $21 \%$ of participants ate meat at least once a day, $63 \%$ of participants ate meat three to four times a week, $12 \%$ ate meat one to four times a month, and $4 \%$ were vegetarians or vegans.

\section{Results}

\section{Model Fit}

The validity of the conjoint model is assessed by observing how well part-worth utilities of the levels can predict the evaluations of the two fixed holdout tasks (Orme et al., 1997). We ran a simulation to estimate participants' choices regarding both holdout
TABLE 4 | Importance of attributes ordered by importance (relative importance values sum to $100 \%$ ).

\begin{tabular}{lr}
\hline Attribute & $\begin{array}{r}\text { Relative attribut } \\
\text { (rounded) }\end{array}$ \\
\hline Weekly meat consumption & 32 \\
Distance to workplace & 22 \\
Means of transport & 13 \\
Room temperature & 12 \\
Holiday travel behavior & 11 \\
Living space & 10
\end{tabular}

tasks based on the individual part-worth utilities derived from the eight random tasks. The simulated results for both holdout tasks were then compared with the actually observed choices regarding the two holdout tasks. A mean absolute error (MAE) test was used to calculate the fitness of the model. This means that, for every holdout task, the difference between the predicted and the observed choices was calculated, with a smaller MAE indicating a better model fit. The MAE for both holdout tasks was 4.24, which is a good result for holdout tasks with three options, according to Orme, President of Sawtooth Software (personal communication). The holdout tasks were only used for this analysis; all further analyses include only the eight randomized tasks.

\section{Importance of Attributes}

All participants who finished the survey were included in the subsequent statistical analysis $(N=150)$. As a first step, the attribute importance values were calculated using Sawtooth Software. Attribute importance is a relative measure that allows a relative comparison among the different attributes used in a study. Relative attribute importance is calculated by dividing the range of part-worth utilities for each attribute by the total utility range for all attributes and multiplying the result by $100 \%$. Therefore, when interpreting importance values, it is important to note that these values are relative to the other attributes in the study and that they depend upon the chosen attribute levels (Orme, 2010). Analyses reveal that meat consumption is the most important attribute for participants, followed by distance to workplace (see Table 4). Means of transport, room temperature, holiday travel behaviors, and living space can be considered less important 
attributes, such that meat consumption is about three times as important as those attributes.

Average part-worth utilities of the levels were calculated and displayed in Figure 1. The part-worth utilities of each attribute sum to 0 . The negative part-worth utilities were not necessarily disliked by participants; however, compared to the other levels, they were preferred less (all else being equal; Orme, 2010). Figure 1 again indicates the greater importance of the two attributes - meat consumption and distance to workplace - but also shows the part-worth utilities represented by the different attribute levels. This allows the preferences for different attribute levels to be identified. Regarding distance to workplace, larger commuting distances $(100 \mathrm{~km})$ were clearly less preferred over shorter commuting distances $(2 \mathrm{~km}$ or $10 \mathrm{~km})$. For commuting, bike and public transport were the most preferred means of transportation, while, for travel behavior, no clear preference pattern emerged. Regarding living space, larger apartments were preferred to smaller ones, and lower room temperatures were preferred to higher temperatures. In terms of food, eating meat daily or several times a week was clearly preferred to a vegetarian diet.

\section{Lifestyles that are Sufficiency-Oriented and Perceived as Being Attractive}

Based on the aggregated part-worth utilities of all the levels, the overall utilities for all composed lifestyles can be calculated (adding up all part-worth utilities for different combinations of attribute levels; in total, $3^{6}=729$ lifestyle combinations are possible). As can be expected based on the results presented in Figure 1, the lifestyle with the highest utility is that of a person who lives around $10 \mathrm{~km}$ from work, commutes to work by bike or public transport, takes holiday trips to Europe, lives in quite a large apartment that is not extensively heated during the winter, and eats meat three to four times a week (first rank, overall utility $=123.49$ ). By contrast, the lowest utility is provided by the following lifestyle: a person who lives around $100 \mathrm{~km}$ from work, commutes to work by car, makes holiday trips to Switzerland, lives in quite a small apartment that is medium heated during the

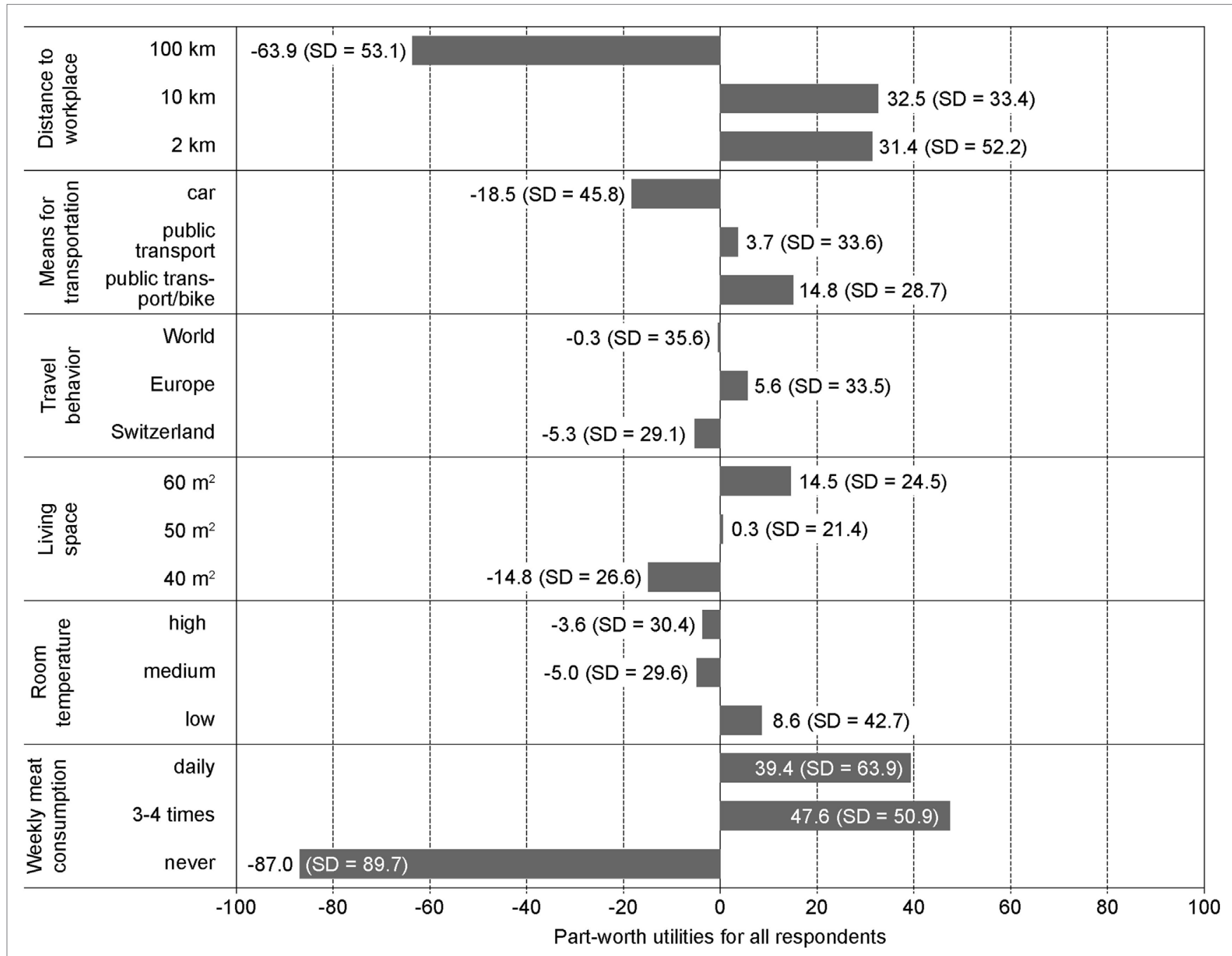

FIGURE 1 | Aggregated average part-worth utilities of all attribute levels. The part-worth attributes for each attribute sum to 0 ( $N=150)$. 
winter and never eats meat (last rank, overall utility $=-194.50$; see Table 5).

A visual inspection of Table 5 indicates several important findings: the 10 most popular lifestyles do not include a strictly vegetarian diet, while the 10 least preferred lifestyles all do. Similarly, the 10 most popular lifestyles are characterized by short commuting distances that are traveled by bike or by car, while the 10 least popular lifestyles are characterized by large commuting distances that are traveled by car. Regarding travel, Switzerland does not seem to be a popular destination, and small apartments are not found among any of the top 10 lifestyles. Regarding room temperature, there is a preference for low temperatures.

In order to make judgments about the sufficiency levels of the various lifestyle concepts, we calculated an additive sufficiency index ( $S$-index) based on the attribute levels (i.e., for the most sufficient level, three points were calculated; for the mid-sufficient level, two points; and for the least sufficient level, one point). We are aware that this is an estimate that does not account for differences in energy-saving potentials among attributes, since all attributes are weighted equally. However, calculating the exact amount of energy per level is difficult, since some of the levels are described quite vaguely (e.g., in the case of the level "Europe" in the domain of travel behavior, the exact number of trips taken is not specified; moreover, the exact destination location within Europe is not specified). Thus, our $S$-index is a very rough estimate of potential savings. It does not reflect differences in energy-saving potentials between domains (e.g., commuting
$2 \mathrm{~km}$ to work is weighted the same as never eating meat), and it gives different weights to levels within domains. The $S$-index takes a value between $S_{\min }=6$ and $S_{\max }=18$, with a higher index representing a more sufficient lifestyle. In Table 5, the $S$-index is displayed for the 10 most preferred and the 10 least preferred lifestyles. On average, the $S$-index for the 10 most preferred lifestyles is slightly higher than the $S$-index for the 10 least preferred lifestyles, indicating that the most preferred lifestyles are not less sufficient than the least preferred ones; rather, the contrary seems to be the case.

The lifestyle composed of the most sufficient attribute levels is represented by a person who lives around $2 \mathrm{~km}$ from work, commutes to work by bike or public transport, makes holiday trips to Switzerland, lives in quite a small apartment that is not heated a lot during winter, and never eats meat. This most sufficient lifestyle is not popular at all - mainly because of its strictly vegetarian diet. It has an overall utility of -52.35 (rank 549 out of 729 ; see Table 6). Thus, the question of interest concerns which sufficiency-oriented lifestyles - specifically, ones that may be less extreme - trigger broad social support and, thus, have the potential to be promising models for energy-sufficient lifestyles for individuals.

Table 6 indicates two key things: first, reasonably energysufficient lifestyles can be found among the 50 most preferred lifestyles (11 of the top 50-ranked lifestyles have $S$-indexes of 14 or $\left.15 ; S_{\max }=18\right)$. However, the most energy-sufficient lifestyles do not seem to be very popular. Second, our results indicate the biggest potentials for support for sufficiency in the domains

TABLE 5 | Ranking of lifestyles (10 top-ranked and lowest-ranked lifestyles), ordered by overall utility.

\begin{tabular}{|c|c|c|c|c|c|c|c|c|c|}
\hline & $\begin{array}{l}\text { Overall } \\
\text { utility }\end{array}$ & Rank & $\begin{array}{c}\text { Distance to } \\
\text { workplace }(\mathbf{k m})\end{array}$ & $\begin{array}{l}\text { Means of } \\
\text { transport }\end{array}$ & $\begin{array}{c}\text { Travel } \\
\text { behavior }\end{array}$ & $\begin{array}{c}\text { Living } \\
\text { space }\left(\mathbf{m}^{2}\right)\end{array}$ & $\begin{array}{c}\text { Room } \\
\text { temperature }\end{array}$ & $\begin{array}{l}\text { Weekly meat } \\
\text { consumption }\end{array}$ & S-index \\
\hline \multirow{10}{*}{$\begin{array}{l}10 \text { most } \\
\text { preferred } \\
\text { lifestyles }\end{array}$} & 123.49 & 1 & 10 & Bike/PT & Europe & 60 & Low & 3-4 times & 13 \\
\hline & 122.47 & 2 & 2 & Bike/PT & Europe & 60 & Low & 3-4 times & 14 \\
\hline & 117.62 & 3 & 10 & Bike/PT & World & 60 & Low & 3-4 times & 12 \\
\hline & 116.60 & 4 & 2 & Bike/PT & World & 60 & Low & 3-4 times & 13 \\
\hline & 115.30 & 5 & 10 & Bike/PT & Europe & 60 & Low & Daily & 12 \\
\hline & 114.28 & 6 & 2 & Bike/PT & Europe & 60 & Low & Daily & 13 \\
\hline & 112.55 & 7 & 10 & Bike/PT & $\mathrm{CH}$ & 60 & Low & 3-4 times & 14 \\
\hline & 112.45 & 8 & 10 & PT & Europe & 60 & Low & 3-4 times & 12 \\
\hline & 111.53 & 9 & 2 & Bike/PT & $\mathrm{CH}$ & 60 & Low & 3-4 times & 15 \\
\hline & 111.43 & 10 & 2 & PT & Europe & 60 & Low & 3-4 times & 13 \\
\hline \multirow{10}{*}{$\begin{array}{l}10 \text { least } \\
\text { preferred } \\
\text { lifestyles }\end{array}$} & -175.87 & 720 & 100 & Car & World & 40 & Low & Never & 12 \\
\hline & -177.99 & 721 & 100 & Car & $\mathrm{CH}$ & 50 & High & Never & 11 \\
\hline & -179.40 & 722 & 100 & Car & $\mathrm{CH}$ & 50 & Medium & Never & 12 \\
\hline & -180.94 & 723 & 100 & Car & $\mathrm{CH}$ & 40 & Low & Never & 14 \\
\hline & -182.15 & 724 & 100 & Car & Europe & 40 & High & Never & 11 \\
\hline & -183.56 & 725 & 100 & Car & Europe & 40 & Medium & Never & 12 \\
\hline & -188.02 & 726 & 100 & Car & World & 40 & High & Never & 10 \\
\hline & -189.43 & 727 & 100 & Car & World & 40 & Medium & Never & 11 \\
\hline & -193.09 & 728 & 100 & Car & $\mathrm{CH}$ & 40 & High & Never & 12 \\
\hline & -194.50 & 729 & 100 & Car & $\mathrm{CH}$ & 40 & Medium & Never & 13 \\
\hline
\end{tabular}

Levels are colored according to sufficiency: white = most sufficient levels; light gray = mid-sufficient levels; dark gray = least sufficient levels. S-Index, sufficiency index, which is calculated by adding points according to level: most sufficient level, 3; mid-sufficient level, 2; least sufficient level, 1. N = 150. 
TABLE 6 | Display of those lifestyles within the 50 most preferred lifestyles with the highest energy sufficiency, ranked by overall utility.

\begin{tabular}{|c|c|c|c|c|c|c|c|c|}
\hline $\begin{array}{l}\text { Overall } \\
\text { utility }\end{array}$ & $\begin{array}{l}\text { Utility } \\
\text { ranking }\end{array}$ & $\begin{array}{c}\text { Distance to } \\
\text { workplace (km) }\end{array}$ & $\begin{array}{l}\text { Means of } \\
\text { transport }\end{array}$ & $\begin{array}{c}\text { Travel } \\
\text { behavior }\end{array}$ & $\begin{array}{c}\text { Living } \\
\text { space }\left(\mathrm{m}^{2}\right)\end{array}$ & $\begin{array}{c}\text { Room } \\
\text { temperature }\end{array}$ & $\begin{array}{l}\text { Weekly meat } \\
\text { consumption }\end{array}$ & $S$-index \\
\hline 122.47 & 2 & 2 & Bike/PT & Europe & 60 & Low & 3-4 times & 14 \\
\hline 112.55 & 7 & 10 & Bike/PT & $\mathrm{CH}$ & 60 & Low & 3-4 times & 14 \\
\hline 111.53 & 9 & 2 & Bike/PT & $\mathrm{CH}$ & 60 & Low & 3-4 times & 15 \\
\hline 109.26 & 15 & 10 & Bike/PT & Europe & 50 & Low & 3-4 times & 14 \\
\hline 108.24 & 18 & 2 & Bike/PT & Europe & 50 & Low & 3-4 times & 15 \\
\hline 103.34 & 27 & 2 & Bike/PT & $\mathrm{CH}$ & 60 & Low & Daily & 14 \\
\hline 102.37 & 31 & 2 & Bike/PT & World & 50 & Low & 3-4 times & 14 \\
\hline 100.49 & 37 & 2 & PT & $\mathrm{CH}$ & 60 & Low & 3-4 times & 14 \\
\hline 100.05 & 40 & 2 & Bike/PT & Europe & 50 & Low & Daily & 14 \\
\hline 98.32 & 46 & 10 & Bike/PT & $\mathrm{CH}$ & 50 & Low & 3-4 times & 15 \\
\hline 97.97 & 48 & 2 & Bike/PT & $\mathrm{CH}$ & 60 & Medium & 3-4 times & 14 \\
\hline 97.30 & 51 & 2 & Bike/PT & $\mathrm{CH}$ & 50 & Low & 3-4 times & 16 \\
\hline 82.20 & 124 & 2 & Bike/PT & $\mathrm{CH}$ & 40 & Low & 3-4 times & 17 \\
\hline-52.35 & 549 & 2 & Bike/PT & $\mathrm{CH}$ & 40 & Low & Never & 18 \\
\hline
\end{tabular}

Below the straight lines, we show the first occurrences of lifestyles with S-Indices of 16, 17, and 18. The last row displays the most sufficient lifestyle (S-index = 18). Levels are colored according to sufficiency: white = most sufficient levels; light gray = mid-sufficient levels; and dark gray = least sufficient levels. $\mathrm{N}=150$.

of distance to workplace, means of transport, and room temperature. By contrast, there did not seem to be any support for lifestyles promoting a strictly vegetarian diet and reduced living space per person.

\section{Discussion}

\section{Key Findings}

This study investigates the societal potentials for sufficiency interventions by investigating people's preferences for different lifestyles using a conjoint analysis. Our first research question is: Which energy-sufficiency-related domains and behaviors do people prefer when evaluating different lifestyles? Based on the data from the conjoint analysis, the following patterns are suggested: distance to workplace and meat consumption are considered to be the most important factors when participants make choices regarding their preferred lifestyle. More specifically, participants strongly preferred shorter commuting distances over longer distances and eating meat several times a week over a vegetarian diet.

Our second research question is: Which energy-sufficient lifestyles are perceived to be attractive by the public? Our data suggest that there are lifestyles that are reasonably energy-sufficient and, at the same time, able to attract broad public support. These lifestyles are characterized by short commuting distances, using bikes and public transport for commuting and lowered room temperatures during the heating season. Lifestyles characterized by a strictly vegetarian diet and reduced living spaces per person were the least preferred ones.

\section{Discussion of Key Findings and Potential Implications}

As demonstrated in our conjoint analysis, there is a disparity between the most energy-sufficient and the most preferred lifestyles. However, this does not mean that energy sufficiency and popular lifestyles must necessarily conflict. There are lifestyles that are both widely preferred and relatively energy-sufficient. Our research has shown that people weigh such domains as mobility, heating, and food differently when making choices about their preferred lifestyles, indicating that they make different trade-offs between these domains. In the following, we separately discuss all of the domains, their potentials for energy sufficiency and possible implications for practice (e.g., for interventions). However, it must be kept in mind that these results were established in an integrated and, thus, indirect way. Also, results are strongly influenced by the attributes and levels chosen and are situated in the context of the German-speaking part of Switzerland.

\section{Shorter Commuting Distances and Mode of Transport}

Our results suggest a preference for shorter commuting distances. This is likely because shorter commuting distances provide significant benefits to individuals in the sense that shorter commutes give employees more leisure time. Similarly, our results suggest a preference for biking to work. This preference may also relate to individual benefits, since "active commuting" (i.e., biking to work) is positively related to physical well-being (Humphreys et al., 2013). Our results reflect a trend in Swiss cities (e.g., Zürich) in which an increasing number of households refrain from having a car, instead opting to use bikes, public transport, or car sharing. In Zürich, $48 \%$ of households do not own a car, and the rate of motorization has declined since the 1990s to around 350 cars per 1000 inhabitants (Stadt Zürich, 2012). This is almost certainly due to the city's excellent public transport system, which offers regular, punctual, and modern means of transport. Our results indicate a high social acceptance for commuting by bike or public transport - and, as such, suggest the potential for interventions to reduce energy consumption through commuting. For example, campaigns that promote biking to work (Bike to Work, 2013), 
sharing offers (e.g., car sharing, bike sharing), or even car-free lifestyles (e.g., car-free residential areas) may be effective. As the level of motorization in the rural areas of Switzerland is still high compared to that in urban areas (Swiss Federal Statistical Office, 2015d), it might be particularly promising to develop respective interventions for rural areas. One interesting example is the bike4car campaign, which encourages car owners to give up driving and try out e-biking for free for a period of 2 weeks ${ }^{3}$. It is important to note that the success of interventions to change commuting behavior depends strongly on the available infrastructure, such as quality of public transport and spatial separation of activities (e.g., shopping, sports, daycare; Thøgersen, 2005). This implies that our results are very context-sensitive and might look very different in another country.

\section{Travel Behavior}

Our study indicates that, relative to the other attributes, holiday travel behavior was not a very important attribute. Our study does not indicate large social support for more local travel behavior; however, this option was perceived as only slightly less attractive than vacations and short trips in Europe or on other continents. We can only speculate about why this might be the case. One reason could be that, although locations varied, all levels seemed to include many short trips and holiday opportunities over the year. Therefore, the different levels might have been perceived as equally attractive, leading only to a small spread across the levels.

\section{Living Space and Room Temperature}

Our study indicates a preference for large living spaces, since $60 \mathrm{~m}^{2}$ per person is clearly preferred to $40 \mathrm{~m}^{2}$ per person. Moreover, our results follow a clear trend in Switzerland toward larger living spaces (Swiss Federal Statistical Office, 2015a). One crucial question is how to address the need for more personal space within cities, where such space is particularly scarce. One option would be to complement a limited amount of personal space with shared spaces (e.g., shared guest rooms, workshops, office spaces, and common rooms). Such shared rooms offer benefits on several different levels: (i) in total, less space needs to be heated, lighted, etc., thus reducing energy consumption; (ii) people can profit from shared infrastructure; and (iii) shared rooms address people's need to connect with other people, in that they offer opportunities to meet, exchange, and learn from each other. Since this approach (i.e., increasing the usage of shared spaces while limiting personal space) addresses different needs and does not only focus on reduced energy consumption, it is particularly promising in terms of attracting broad social support (International Energy Agency, 2014; Moser et al., 2014). Regarding room temperature, the study indicates a certain potential for interventions to reduce room temperature, since lower room temperatures were preferred to higher temperatures. However, it should be noted here that the study was conducted in autumn, before the heating season; thus, the results may be framed by the time of year. Interestingly, the results of our study indicate paradoxical effects regarding living space and room temperature that are similar to those determined by Sütterlin and

${ }^{3}$ www.bike4car.ch
Siegrist (2014). They found that participants regard a person who has more living space but lower room temperatures to be more environmentally conscious than a person with less living space but higher room temperatures, although the latter actually uses less energy for heating.

\section{Meat Consumption}

Our results clearly show that a vegetarian or vegan diet is not a viable option for the vast majority of participants. For most participants, it was important to eat meat several times per week or even daily. However, the results also showed that daily meat consumption is not the most preferred option, indicating that many people are ready to refrain from meat consumption from time to time. A link to personal health may play an important role here. Based on our results, we may speculate that restrictions on eating meat might trigger strong reactions and protests. Instead, campaigns promoting vegetarian dishes from time to time may be more effective. Furthermore, people might be nudged into less energy-intense dietary habits through attractive alternatives in canteens and restaurants (Bucher et al., 2011).

\section{Critical Reflections, Limitations and Further Research}

In our study, we see three particular limitations related to: the choice of levels, context influences, and the construction of the $S$-index.

In conjoint analyses, the importance values of attributes are vastly influenced by the levels and the spread associated with the attributes. Although Orme (2002) suggests that levels should spread across the full range of possibilities, this might have been too extreme for the meat consumption attribute. As shown in Table 4 and Figure 1, the importance of the meat consumption attribute was significantly influenced by the negative part-worth utility of the level of vegetarian diet (never). In comparison with the other levels (i.e., daily meat consumption and three to four times meat consumption per week), this option is more extreme because it suggests a strictly vegetarian or vegan diet. It is possible that a less extreme level (e.g., meat consumption once a month) would not have triggered such extreme reactions by participants. On the other hand, our responses are in line with the intense public response that followed the proposal by some Swiss canteens to launch "vegetarian days." This announcement resulted in large protests in social media and through online comments to media articles, indicating that meat consumption may be nonnegotiable for many Swiss people. Although daily consumption is not necessarily desired, many people in Switzerland are not ready to completely give up meat consumption. In 2012, only around $3 \%$ of Switzerland's population never ate meat or sausage products (Swiss Federal Office of Public Health, 2014). For a future study, it would be interesting to add another level between never and three to four times a week, such as once a month or on special occasions. A similar logic regarding the spread of levels can be found in the attribute commuting distance, since the commuting distances of 2 and $10 \mathrm{~km}$ are quite close together, while $100 \mathrm{~km}$ is more extreme. It would be interesting to include a less extreme option in a future study, thereby allowing the identification of tipping points in preferences with respect to commuting distances. 
It is likely that our study results are affected by contextual influences. These influences may have manifested, in particular, in participant responses to the attribute room temperature. Here, many participants preferred lifestyles in which the protagonists wore warm clothes during winter to keep warm in their apartments, instead of turning up the thermostat. This result may have been influenced by contextual effects, since the data were collected in September and not during the heating season. Furthermore, the amount of clothing worn serves only as a proxy for drawing conclusions about the actual room temperature (in terms of absolute values). It could be that people prefer warm clothing, but simultaneously heat their living spaces to higher temperatures. However, we would argue that it is easier for participants to imagine the types of clothes implied by room temperature than to imagine a particular room temperature in degree celsius. Similarly, our results regarding travel behavior could be influenced by the season in which the study was conducted. If the study had been conducted before summer, when many people in Switzerland usually plan longer vacations, preferences for flying to distant countries might have been more distinct. In general, actual behaviors cannot be inferred directly from the revealed preferences, as there might be additional constraints in people's lives. For example, although a participant might prefer a commuting distance of $2 \mathrm{~km}$, he or she may not be able to move closer to the workplace or change jobs due to his or her family situation.

Although our $S$-index provides certain indications regarding the energy-saving potentials of the presented lifestyles, these indications offer only a very rough estimate, which does not account for differences in the potential savings of the attributes. For future research, it would be interesting to describe the levels more precisely. More finely grained data would facilitate the calculation of the actual energy-saving potentials of the presented lifestyles (e.g., based Life Cycle Assessment databases), thus allowing us to draw precise conclusions about the energy-saving potentials of the most preferred lifestyles.

\section{Conclusion}

Our study results suggest that the success of energy-sufficiency interventions might depend strongly on the targeted behavior.

\section{References}

Abrahamse, W., Steg, L., Vlek, C., and Rothengatter, T. (2005). A review of intervention studies aimed at household energy conservation. J. Environ. Psychol. 25, 273-291. doi:10.1016/j.jenvp.2005.08.002

Barr, S., Gilg, A., and Shaw, G. (2011). "Helping people make better choices": exploring the behaviour change agenda for environmental sustainability. Appl. Geogr. 31, 712-720. doi:10.1016/j.apgeog.2010.12.003

Bike to Work. (2013). Ergebnisse Befragung TeilnehmerInnen, TeamchefInnen [Survey Results of Teamleaders and Participants]. Bern: Bike to Work.

Breukers, S., Mourik, R., and Heiskanen, E. (2013). "Changing energy demand behavior: potential of demand-side-management," in Handbook of Sustainable Engineering, eds Kauffmann J. and Lee K.-M. (Dordrecht: Springer), 773-792.

Bucher, T., van der Horst, K., and Siegrist, M. (2011). Improvement of meal composition by vegetable variety. Public Health Nutr. 14, 1357-1363. doi:10.1017/ S136898001100067X

Bügl, R., Stauffacher, M., Kriese, U., Pollheimer, D. L., and Scholz, R. W. (2012). Identifying stakeholders' views on sustainable urban transition: desirability,
Interventions to change certain behaviors (e.g., meat consumption) seem likely to face strong public resistance. As such, our results have implications for the promotion of energy-sufficient lifestyles through, for example, energy-saving campaigns. Specifically, our results show that extremely energy-sufficient lifestyles are not perceived as attractive - or, more technically speaking, they are characterized by a negative overall utility. We thus speculate that the promotion of such extremely energy-sufficient lifestyles might backfire, potentially evoking resistance or resignation.

Our study results could be interpreted to suggest that wellbalanced lifestyles with substantive (but not extreme) energysaving potentials might better serve as social models for energy sufficiency (compared to extremely sufficient lifestyles). As role models, such well-balanced lifestyles may motivate people to change their routine behaviors and lifestyles in order to consume less energy. A similar effect was found in a study on scenarios for urban development, in which the most sustainable scenarios were unable to trigger consensus among different stakeholders (e.g., investors, urban planners, housing target groups), whereas more balanced scenarios were able to gain broader support (Bügl et al., 2012). However, the study at hand is exploratory; thus, questions concerning exactly how the public reacts when confronted with extreme energy-sufficient lifestyles or respective interventions, what types of emotions these lifestyles trigger and how well different groups identify with them remain unanswered. Field experiments could be a promising approach to investigate these questions.

\section{Acknowledgments}

This paper is based on the MSc thesis of the second author, supervised by the first and third author. The work was supported by the National Research Programme "Managing Energy Consumption" (NRP 71) of the Swiss National Science Foundation (SNSF). Further information on the National Research Programme can be found at www.nrp71.ch. According to ETH ethical guidelines, no ethical approval was required for this study. We would like to thank the participants for taking part in the study. Furthermore, we would like to thank Yann Blumer, Merla Kubli, the two reviewers Anya Skatova and Andrea Tabi for providing feedback on earlier versions of this paper, as well as Sandro Bösch for designing Figure 1.

utility and probability assessments of scenarios. Eur. Plann. Stud. 20, 1667-1687. doi:10.1080/09654313.2012.713332

Darby, S. (2007). "Enough is as good as a feast: sufficiency as policy," in ECEEE 2007 Summer Study, 111-119.

Dohle, S., Keller, C., and Siegrist, M. (2010). Conjoint measurement of base station siting preferences. Hum. Ecol. Risk Assess. 16, 825-836. doi:10.1080/10807039 .2010 .501250

Dutilh, C. E., and Kramer, K. J. (2000). Energy consumption in the food chain. Ambio 29, 98-101. doi:10.1579/0044-7447-29.2.98

Gardner, G. T., and Stern, P. C. (2008). The short list: the most effective actions US households can take to curb climate change. Environment 50, 12-24. doi:10.3200/envt.50.5.12-25

Green, P. E., and Srinivasan, V. (1978). Conjoint analysis in consumer research: issues and outlook. J. Consum. Res. 5, 103-123. doi:10.1086/208721

Herring, H. (2006). Energy efficiency: a critical view. Energy 31, 10-20. doi:10.1016/j.energy.2004.04.055

Higginson, S., Thomson, M., and Bhamra, T. (2014). "For the times they are a-changin": the impact of shifting energy-use practices in time and space. Local Environ. 19, 520-538. doi:10.1080/13549839.2013.802459 
Huddart Kennedy, E., Krahn, H., and Krogman, N. T. (2015). Are we counting what counts? A closer look at environmental concern, pro-environmental behaviour, and carbon footprint. Local Environ. 20, 220-236. doi:10.1080/13 549839.2013.837039

Humphreys, D. K., Goodman, A., and Ogilvie, D. (2013). Associations between active commuting and physical and mental wellbeing. Prev. Med. 57, 135-139. doi:10.1016/j.ypmed.2013.04.008

International Energy Agency. (2014). Capturing the Multiple Benefits of Energy Efficiency. Paris: OECD/IEA.

Jenny, A. (2014). Suffizienz auf individueller Ebene - Literaturanalyse zu psychologischen Grundlagen der Suffizienz. Zwischenbericht Nr. 18, Forschungsprojekt FP-1.7. Zürich: Energieforschung Stadt Zürich.

Jungbluth, N., and Itten, R. (2012). Umweltbelastungen des Konsums in der Schweiz und in der Stadt Zürich: Grundlagendaten und Reduktionspotenziale. Zürich: Energieforschung Stadt Zürich.

Karlin, B., Davis, N., Sanguinetti, A., Gamble, K., Kirkby, D., and Stokols, D. (2012). Dimensions of conservation: exploring differences among energy behaviors. Environ. Behav. 46, 423-452. doi:10.1177/0013916512467532

Krütli, P., Stauffacher, M., Pedolin, D., Moser, C., and Scholz, R. W. (2012). The process matters: fairness in repository siting for nuclear waste. Soc. Justice Res. 25, 79-101. doi:10.1007/s11211-012-0147-x

Linz, M. (2012). Weder Mangel noch Übermass: Warum Suffizienz unentbehrlich ist. München: Oekom Verlag.

Linz, M., Bartelmus, P., Hennicke, P., Jungkeit, R., Sachs, W., Scherhorn, G., et al. (2002). "Von nichts zu viel: Suffizienz gehört zur Zukunftsfähigkeit," in Wuppertal Working Papers, Wuppertal: Wuppertal Institut, 125.

Moser, C., von Wirth, T., Adler, C., Fonseca, J., Schlüter, A., and Stauffacher, M. (2014). Nachhaltige Arealentwicklung: Der Fall Siemensareal in Zug [Sustainable Urban Development: The Case of the Siemens Site in Zug]. Zürich: ETH-UNS TdLab.

Mourik, R., and Rotmann, S. (2013). Most of the Time What We Do is What We Do Most of the Time. And Sometimes We Do Something New: Analysis of Case Studies IEA DSM Task 24 Closing the Loop - Behaviour Change in DSM: From Theory to Practice. Paris: International Energy Agency.

Notter, D. A., Meyer, R., and Althaus, H.-J. (2013). The western lifestyle and its long way to sustainability. Environ. Sci. Technol. 47, 4014-4021. doi:10.1021/ es3037548

Orme, B. K. (2002). Formulating Attributes and Levels in Conjoint Analysis. Sequim, WA: Sawtooth Software.

Orme, B. K. (2010). Getting Started with Conjoint Analysis: Strategies for Product Design and Pricing Research, 2nd Edn. Madison, WI: Research Publishers LLC.

Orme, B. K., Alpert, M. I., and Christensen, E. (1997). Assessing the Validity of Conjoint Analysis - Continued, Vol. 98382. Sequim, WA: Sawtooth Software.

Owens, S., and Driffill, L. (2008). How to change attitudes and behaviours in the context of energy. Energy Policy 36, 4412-4418. doi:10.1016/j.enpol.2008.09.031

Poortinga, W., Steg, L., Vlek, C., and Wiersma, G. (2003). Household preferences for energy-saving measures: a conjoint analysis. J. Econ. Psychol. 24, 49-64. doi:10.1016/s0167-4870(02)00154-x

Rösch, A. (2013). Analysis of Energy Sufficiency: Barriers and Opportunities on the Path Towards an Energy Sufficient Consumer Society. Zürich: ETH Zürich.

Rudolf, M., Seidl, R., Moser, C., Krütli, P., and Stauffacher, M. (2014). Public preferences of electricity options before and after Fukushima. J. Integr. Environ. Sci. 11, 1-15. doi:10.1080/1943815X.2014.881887

Sattler, H., and Hensel-Börner, S. (2001). "A comparison of conjoint measurement with self-explicated approaches," in Conjoint Measurement, eds Gustafsson A., Herrmann A., and Huber F. (Berlin: Springer), 121-133.

Sawtooth Software. (2008). CBCv6.0 Technical Paper. Orem: Sawtooth Software, Inc. Stadt Zürich. (2006). Raumtemperatur-Richtlinie. Zürich: Stadt Zürich.

Stadt Zürich. (2012). Mobilität in Zahlen [Mobility Figures]. Zürich: Stadt Zürich.
Steg, L., and Vlek, C. (2009). Encouraging pro-environmental behaviour: an integrative review and research agenda. J. Environ. Psychol. 29, 309-317. doi:10.1016/j.jenvp.2008.10.004

Sütterlin, B., Brunner, T. A., and Siegrist, M. (2011). Who puts the most energy into energy conservation? A segmentation of energy consumers based on energy-related behavioral characteristics. Energy Policy 39, 8137-8152. doi:10.1016/j. enpol.2011.10.008

Sütterlin, B., and Siegrist, M. (2014). The reliance on symbolically significant behavioral attributes when judging energy consumption behaviors. J. Environ. Psychol. 40, 259-272. doi:10.1016/j.jenvp.2014.07.005

Swiss Federal Council. (2013). Botschaft zum ersten Massnahmenpaket der Energiestrategie 2050 und zur Volksinitiative «Für den geordneten Ausstieg aus der Atomenergie (Atomausstiegsinitiative)». Bern: Swiss Federal Council.

Swiss Federal Office of Energy. (2013). Analyse des schweizerischen Energieverbrauchs 2000-2012 nach Verwendungszwecken [Energy Consumption in Switzerland According to Purpose 2000-2012]. Bern: Federal Department of the Environment, Transport, Energy and Communications.

Swiss Federal Office of Energy. (2014). Schweizerische Gesamtenergiestatistik 2013 [Swiss Energy Statistics]. Ittigen: Swiss Federal Office of Energy.

Swiss Federal Office of Public Health. (2014). Schweizerische Gesundheitsbefragung 2012: Standardtabellen [Swiss Health Survey 2012: Tables]. Available at: http:// www.portal-stat.admin.ch/sgb2012/

Swiss Federal Statistical Office. (2012). Taschenstatistik der Schweiz 2012 ["Pocket Statistics" Switzerland 2012]. Neuchâtel: Swiss Federal Statistical Office.

Swiss Federal Statistical Office. (2013a). Lebenslanges Lernen in der Schweiz: Ergebnisse des Mikrozensus Aus- und Weiterbildung 2011 [Lifelong Learning in Switzerland: Results from the Education Census 2011]. Neuchâtel: Swiss Federal Statistical Office.

Swiss Federal Statistical Office. (2013b). Reisen der Schweizer Wohnbevölkerung 2012 [Trips of Swiss People 2012]. Neuchâtel: Swiss Federal Statistical Office.

Swiss Federal Statistical Office. (2014). Pendlermobilität in der Schweiz: 2012 [Commuting in Switzerland: 2012]. Neuchâtel: Swiss Federal Statistical Office.

Swiss Federal Statistical Office. (2015a). Bau- und Wohnungswesen: Die wichtigsten Zahlen [Construction and Housing: Key Figures]. Available at: http://www.bfs. admin.ch/bfs/portal/de/index/themen/09/01/key.html

Swiss Federal Statistical Office. (2015b). Families, Households - Data, Indicators: Households by Household Size 2013. Available at: http://www.bfs.admin.ch/bfs/ portal/en/index/themen/01/04/blank/key/01/05.html

Swiss Federal Statistical Office. (2015c). Population: Key Figures 2013. Available at: http://www.bfs.admin.ch/bfs/portal/en/index/themen/01/01/key.html

Swiss Federal Statistical Office. (2015d). Transport Infrastructure and Vehicles - Vehicles Stock, Level of Motorisation. Available at: http://www.bfs. admin.ch/bfs/portal/en/index/themen/11/03/blank/02/01/01.html

Thøgersen, J. (2005). How may consumer policy empower consumers for sustainable lifestyles? J. Consum. Policy 28, 143-177. doi:10.1007/s10603-005-2982-8

Urban, J., and Ščasný, M. (2012). Exploring domestic energy-saving: the role of environmental concern and background variables. Energy Policy 47, 69-80. doi:10.1016/j.enpol.2012.04.018

Conflict of Interest Statement: The authors declare that the research was conducted in the absence of any commercial or financial relationships that could be construed as a potential conflict of interest.

Copyright (c) 2015 Moser, Rösch and Stauffacher. This is an open-access article distributed under the terms of the Creative Commons Attribution License (CC BY). The use, distribution or reproduction in other forums is permitted, provided the original author(s) or licensor are credited and that the original publication in this journal is cited, in accordance with accepted academic practice. No use, distribution or reproduction is permitted which does not comply with these terms. 